\title{
Design and implementation of embedded auto car parking system using FPGA for emergency conditions
}

\author{
Khor Jing Yong, Muataz H. Salih \\ School of Computer and Communication Engineering, Universiti Malaysia Perlis (UniMAP), Malaysia
}

\begin{tabular}{l} 
Article Info \\
\hline Article history: \\
Received Sep 23, 2018 \\
Revised Nov 5, 2018 \\
Accepted Dec 28, 2018 \\
\hline
\end{tabular}

\section{Keywords:}

Automatic car parking

Embedded system

FPGA system design

sensors

Smart car

\begin{abstract}
An automatic car parking system using FPGA based on emergency conditions was proposed to detect the driver's condition and perform specific tasks such as warn the drivers and automatic parking depends on the behavior of the driver. Long journey driving on highway will lead loss of concentration of driver quickly and also cause the driver doze off. Based on statistics, driver tends to fall asleep more on high-speed, long, boring highway. Most crashes occur between 4.00a.m-6.00a.m and midnight 12.00a.m till 2.00a.m. Besides, heart attack during driving will also harm to other road users. The risk of accident will increase due to these conditions. In this paper, automatic car parking system using FPGA based on emergency conditions was proposed to detect the driver's condition and perform specific tasks such as warn the drivers and automatic parking depends on the behavior of the driver. At the same times, this system can avoid all the obstacles and cars on the road from time to time. Input data management unit was designed as control unit for input data sensors of input and output control. Main processing was designed as speed controller and also the steering controller. Speed controller used to control the speed of vehicle while driving on the road by detecting the obstacles. Steering controller was designed to control automatic car parking and also assisted with sensors around the vehicles. Sensors were tested with many materials so that the system can flexible with any conditions. The total of 1083 logic elements and 676 registers being used in this project and up to $1.6 \mathrm{GHz}$ as operating frequency. The complete design is able to avoid obstacles surrounding of host vehicles and parked at a safe area automatically after heart attack of driver detected. Then, GSM send GPS coordination to hospital.
\end{abstract}

Copyright @ 2019 Institute of Advanced Engineering and Science. All rights reserved.

Corresponding Author:

Muataz Hameed Salih,

School of Computer and Communication Engineering,

Universiti Malaysia Perlis (UniMAP),

Level 1 Pauh Compus, 02600 Arau, Perlis, Malaysia.

Email: muatazhameed@gmail.com

\section{INTRODUCTION}

Nowadays, current Driver Assistance System (DAS) was designed for comfortable and safety driving [1]. The most popular DAS is the smart car parking system by using sensors to detect surrounding car. However, there is a lot of improvement for DAS including cruise control, automatic navigation system, lane departure warning, etc. [2]. Long journey driving on highway will lead loss of concentration of driver quickly and also cause the driver doze off. Besides, heart attack during driving will also harm to other road users [3]. The risk of accident will increase due to these conditions. Therefore, this system will be designed to detect the driver's condition and perform specific tasks such as warn the drivers and automatic parking depends on the behavior of the driver. At the same times, this system can avoid all the obstacles and cars on the road from time to time $[4,5]$. 
Recent paper and research have focused on the driver assistance system. The comparison for various medical diagnose instrument like EEG, ECG, GSR and PPG for drowsiness detection is proposed by many researchers [6-11]. In-ear EEG has the highest accuracy to detect drowsiness if compared to GSR, PPG and ECG. The torque concept is implemented into steering torque control to prevent lane departure also investigated $[12,13]$. This method definitely will be one of approach of advance driver assistance system in the future. The combination of laser range finder and camera by using HOG and SVM is implemented to reduce the number of computational processes of captured image and makes the system more efficient. Besides, this will increase the speed of the system in order to prevent host vehicles from crashing [14-16].

There is several electronic components used for efficient monitoring and also vehicle control including infrared sensors, heart beat detector, GPS, GSM and Arduino Uno R3 [16]. De1-SoC which is FPGA plays important roles as control unit and main processing unit. De1-SoC consists of 8 channel of ADC at a rate of $500 \mathrm{KSPS}$ to receive the input sensors data. All the input data sensors will be processed concurrently. Main processing unit will control the speed vehicles due to the distance of host vehicle and the obstacles on the road. Infrared sensors used to measure distances between host vehicles and obstacles. Heart beat detector used to detect the healthy problem of driver. GPS and GSM works together with Arduino. GPS used for localization and GSM will send the coordinate of host vehicles of hospital when there is a healthy problem with drivers after the car parked at a safe area. However, Arduino acts like an off chip module.

\section{RESEARCH METHOD}

According to literature review, driver assistance system plays an important role in usual life for comfortable driving environment and safety of driver. Current driver assistance system is designed with several features including automatic navigation system, cruise control, automatic parking, lane departure warning, lane change assist, etc. However, most the current driver assistance system that has been designed did not reduce the most of accident cause which is drowsiness of driver and sudden heart attack. Therefore, an automatic car parking system using FPGA based on emergency condition will be designed to enhance the current driver assistance system. In addition, this project based FPGA using DE1-SoC have high speed data processing and lower consumption.

De1-SoC board is one of the FPGA development platforms which commonly used for project of variety sensors. This is because of DE1-SoC consists of 8 channel of ADC at a rate of $500 \mathrm{KSPS}$ to receive multiple input sensors data. Besides, it also consists of 64MB SDRAM on FPGA and 1GB DDR3 SDRAM ON HPS. It is suitable for embedded soft processors with $85 \mathrm{~K}$ programmable logic elements.

\subsection{Top Level Design}

Top level design is an overview of a system which will describe the operation for each of the functional block. The top level design diagram for this system consists of input manager input, capture logic, ADC controller, main processing unit and others as shown in Figure 1. This kind of design divides the entire system into smaller parts and more easily to determine the connectivity between all the components.

\subsection{Sensors Testing}

There are various sensors in this project including ultrasonic sensor and ECG sensors. Sensors of this project are detected heartbeat and distance. Testing procedure is necessary to all sensors to make sure the functionality of sensor. Output of sensors usually is electronic signal which is voltage but also could be current, frequency and phase. Digital storage oscilloscope (DSO) is one of the best instruments to observe, measure and troubleshoot the electrical signal which produced by sensors. Testing procedure is necessary to make sure the detection of real world parameters by sensors is accurate with the electrical signal which is understandable by system itself. Signal conditioning circuit is needed to improve stability and quality of system by indicate and storing the signal which produced by sensors.

\subsection{ADC Controller}

ADC stands for analog-digital converter and converts the analog signals from sensors such as distance estimated by infrared sensors and heart beat detector into digital signals which are understandable by most of the processors. The analog signals are usually in measurement of voltage and current. ADC converted this measurement to digital number which is usually in binary number. For infrared sensors, analog voltage output reading of ultrasonic sensors will get larger as the distance between targets with sensors increases. 


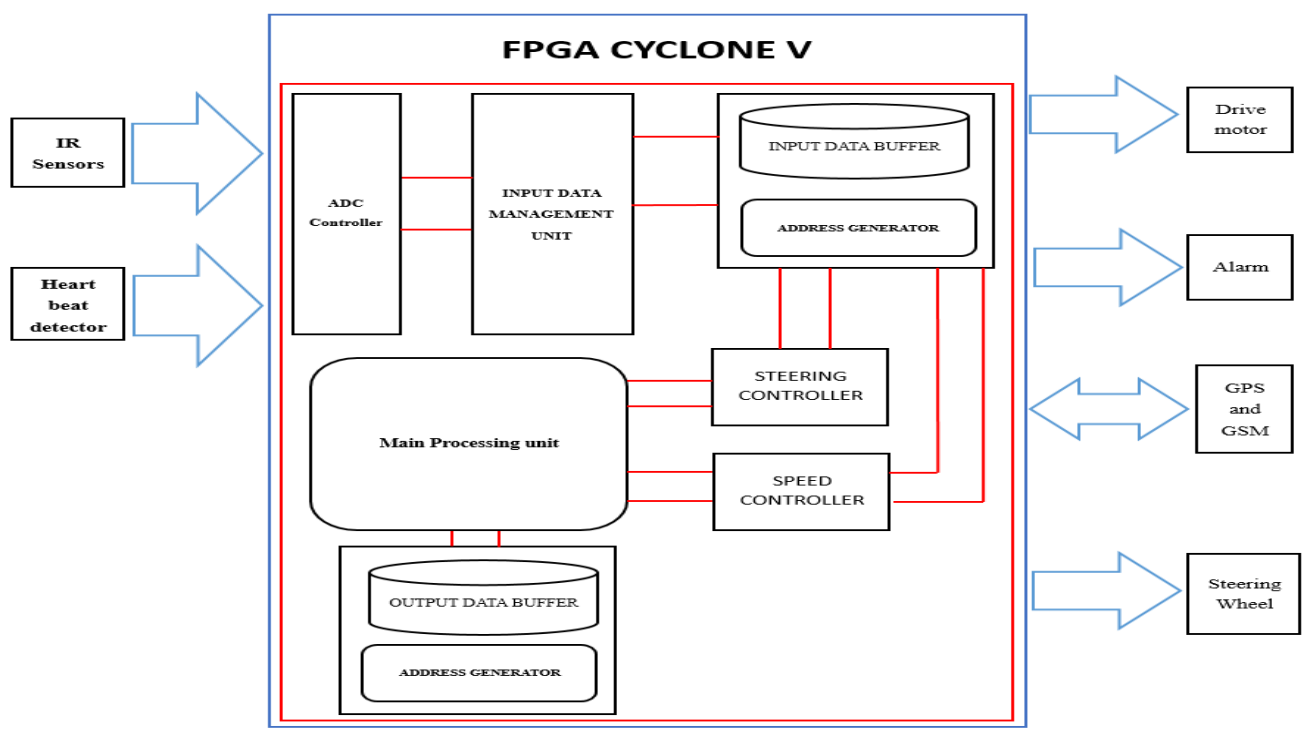

Figure 1. Top level design of embedded automatic car parking on FPGA based for emergency condition

\subsection{Input Data Management Unit}

Input data management unit act as a central unit between ADC controller and main processing unit. Main purpose of input data management unit is to analyze the ECG signal from driver during high speed driving. ECG signal consists of different segment which provide sufficient information about heart failure and also heart attack. Besides, all the electrical signal from different sensors have different format, frequency and transfer speed will be processed in input data management unit Synchronization process will be carried out in input data management unit towards different input data from different sensors which transferred from $\mathrm{ADC}$ controller. The signal will be transferred to main processing unit once the synchronization process was finished. As conclusion, input data management unit receive the entire signal from ADC controller and transfer those signal to main processing unit once synchronization process was finished.

\subsection{Main Processing Unit}

Main processing unit is like a core of entire system. It consists of 3 main modules which is steering controller, speed controller and also ECG analyzer. Steering controller used to control steering when the system is at automatic car parking mode. Speed controller is to control the speed of vehicle due to the distances of obstacle. ECG analyzer is to analyze and encode the ECG signal from driver into different pulsing signal by detecting driver's behavior and eventually able to detect heart condition of driver. Main processing unit collect all input from each of the on-chip module like ADC controller and then generate output which sent to different devices. Main processing unit will carry out all the instruction by performing arithmetic, logical, control and input/output operations. First stage, main processing unit collects all the data of different on chip module from input data buffer. For second stage, it will interprets the input data and execute specific computational for each of input data like counters, arithmetical, logical and control operations. After computational is finished, main processing unit will store the output data into output data buffer which ready to be transferred to output devices.

Pipelining technique used in this project. High throughput of system and less consumption power is always the common needs for a system. Parallel computing is definitely one of the computational which commonly used in FPGA-based system. Spatial parallelism used since it can duplicate hardware which can perform multiple tasks at once. Unlike temporal parallelism which is the task is broken into multiple stages. The main objectives of this project are a system based high performance; tasks executed concurrently and lower consumption power. Spatial parallelism proposed as the method which can meet the requirement of system.

The main processing units designed using the concept of spatial parallelism. In this project, the number of main processing unit will be determined by the number of tasks. This means all main processing units will execute concurrently at the same time without delaying any input data to be processed especially in speed control, steering control and also ECG analyzer. These specific tasks must be executed at the same time. All the main processing units will be controlled by a clock to make sure the tasks execute at the same time. 


\section{RESULTS AND ANALYSIS}

\subsection{Sensor Testing Results}

For infrared sensors, analog voltage output reading of ultrasonic sensors will get larger as the distance between targets and sensors increases. All the infrared sensors tested with different color and material of the obstacle including white, red, black, and semitransparent as shown in Figure 2 and 3.

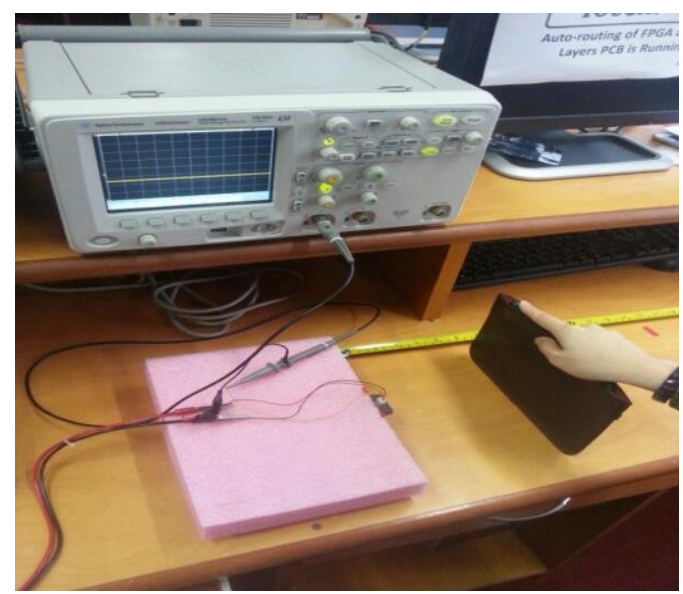

Figure 2. IR Sensor testing with black color

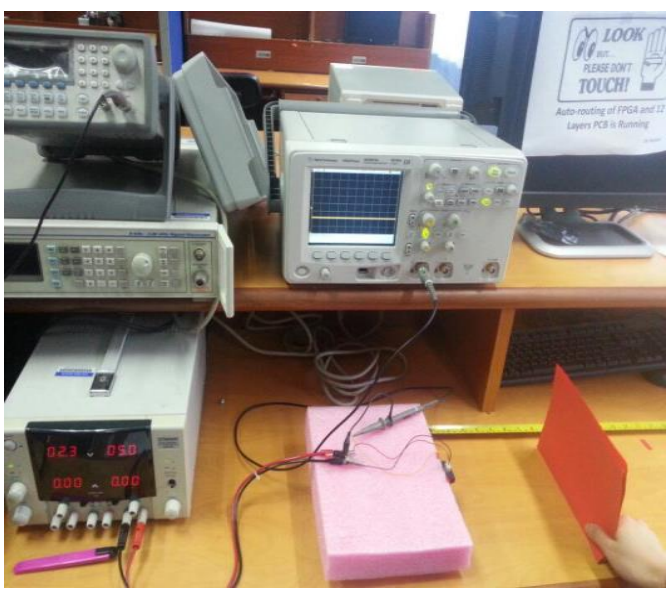

Figure 3. IR Sensor testing with red color

From the Table 1, the sensitivity of IR sensors is around with the distance of $40 \mathrm{~cm}$. The threshold of IR sensors is at $3 \mathrm{~cm}$ which can be seen obviously in the table. The semitransparent has lowest reflectivity among all the objects and offset output voltage of semitransparent object is around 2.5V.There are several factor that affecting data IR sensor including voltage supply, light condition, surrounding obstacles and its own reflectivity.

At the same time, Quartus II software In system memory editor used to test input data sensor. This is to make sure the accuracy of data and also the safety of the system. In the testing process, there is a lot of unstable of sensor data. For example, every five readings taken are followed by one unstable reading. This pattern is not always being the same and it might be every eight readings followed by one unstable reading as shown in Figure 4. A stabilize data module designed to synchronize the unstable data and also different frequency among different modules. Figures 5,6 and 7 show the IR sensors location on the car. Also, Figure 8 shows the ECG sensor on car to detect driver heartbeat attack and activate auto parking system process.

Table 1. The IR Sensor Testing Result (voltage)

\begin{tabular}{ccccc}
\hline Distance & White & Red & Black & Semi transparent \\
\hline 0 & 0.0000 & 0.0000 & 0.0000 & 0.0000 \\
1 & 1.2500 & 1.1250 & 0.1250 & 2.5000 \\
3 & 2.8750 & 2.8750 & 2.8125 & 2.5000 \\
5 & 2.2500 & 2.1250 & 2.2500 & 1.6875 \\
7 & 1.6875 & 1.6875 & 1.6250 & 1.3125 \\
9 & 1.3125 & 1.3125 & 1.2500 & 1.0000 \\
10 & 1.1875 & 1.1250 & 1.1875 & 0.9375 \\
12 & 1.0000 & 1.0000 & 0.9375 & 0.7500 \\
15 & 0.8125 & 0.7500 & 0.6875 & 0.6250 \\
20 & 0.6250 & 0.6250 & 0.5000 & 0.3750 \\
25 & 0.5000 & 0.5000 & 0.3750 & 0.3125 \\
30 & 0.3750 & 0.3750 & 0.3125 & 0.2500 \\
35 & 0.3125 & 0.3125 & 0.2500 & 0.1875 \\
40 & 0.2500 & 0.2500 & 0.2500 & 0.1875 \\
\hline
\end{tabular}




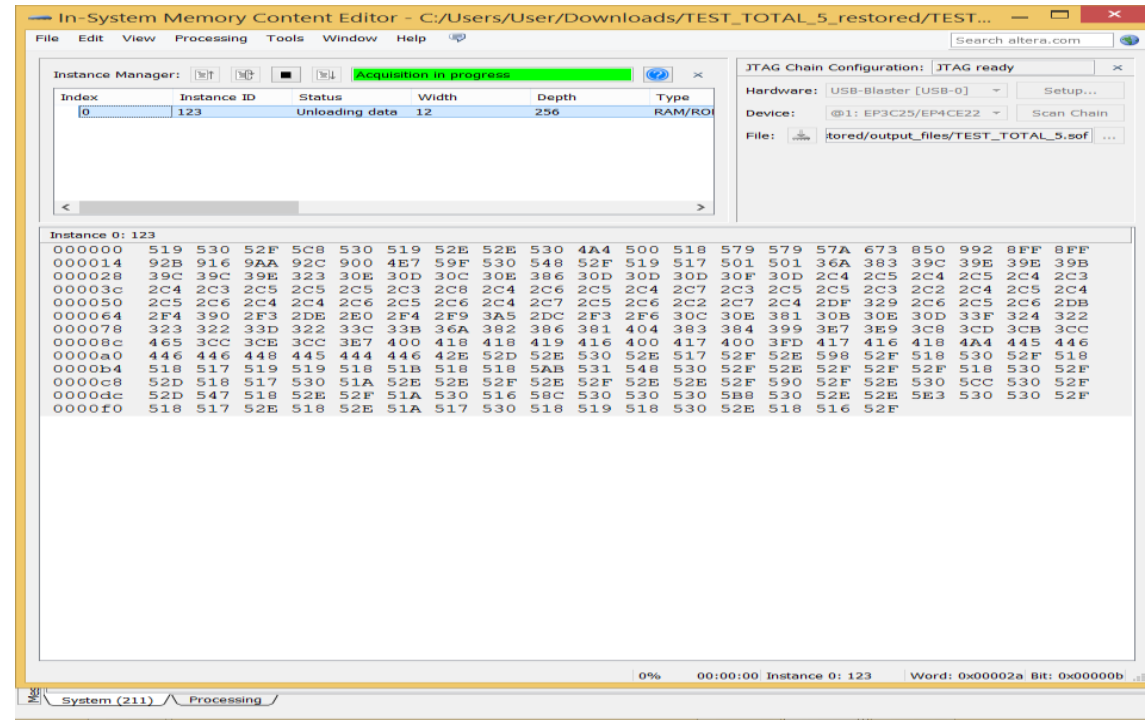

Figure 4. In system memory heart beat and IR sensor data

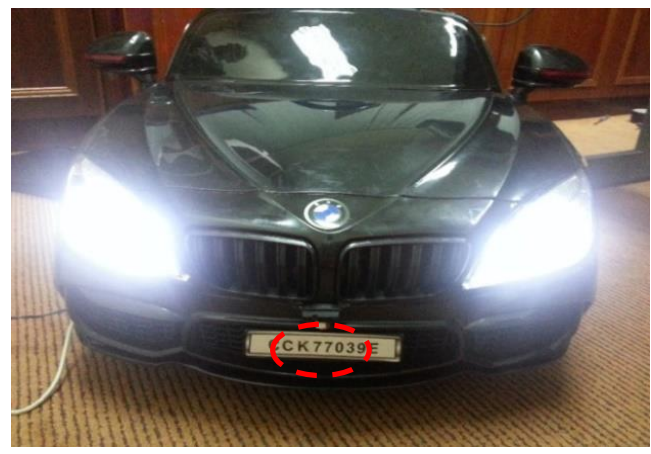

Figure 5. IR sensor front view on vehicle

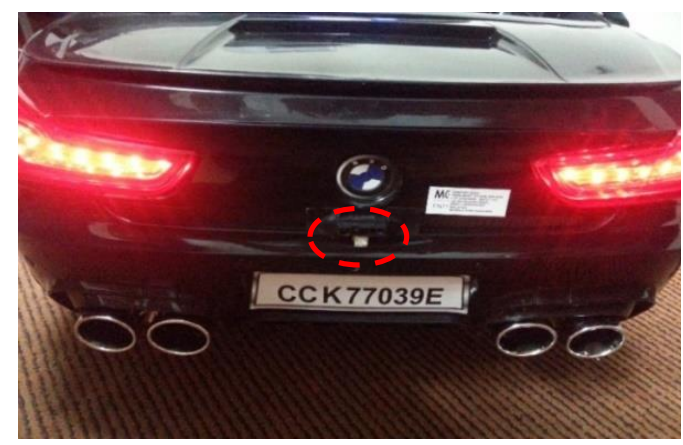

Figure 7. IR sensor back view on vehicle

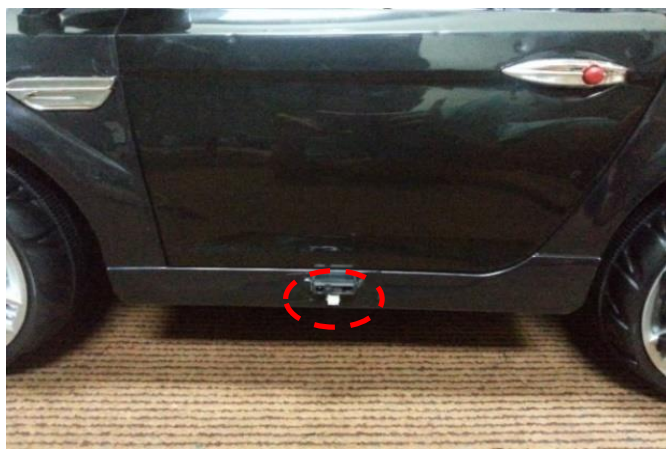

Figure 6. IR sensor side view on vehicle

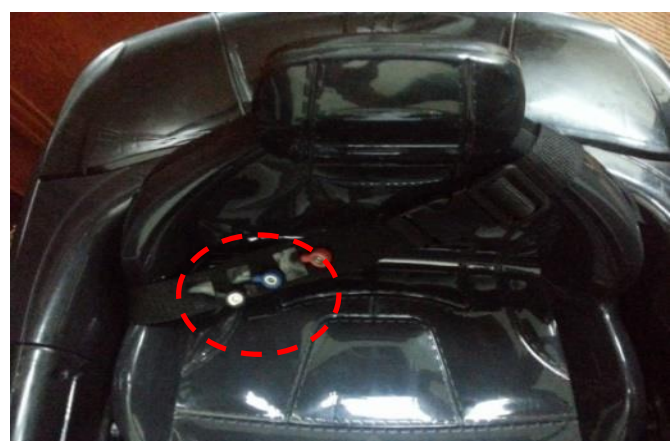

Figure 8. ECG sensor on car

\subsection{ECG Signal Analyses}

Figure 9 shows a standard cycle of ECG signal. There is $\mathrm{P}$ wave, QRS wave and $\mathrm{T}$ wave. $\mathrm{PR}$ interval is the time interval for electrical pulse transmitted from atria to ventricles. Heart failure such as fibrillation and arrhythmias information can found in QRS segment. ST segment can provide the information of insufficient blood supply to organ especially heart muscles. The T wave in QT interval could provide information about high blood pressure. 


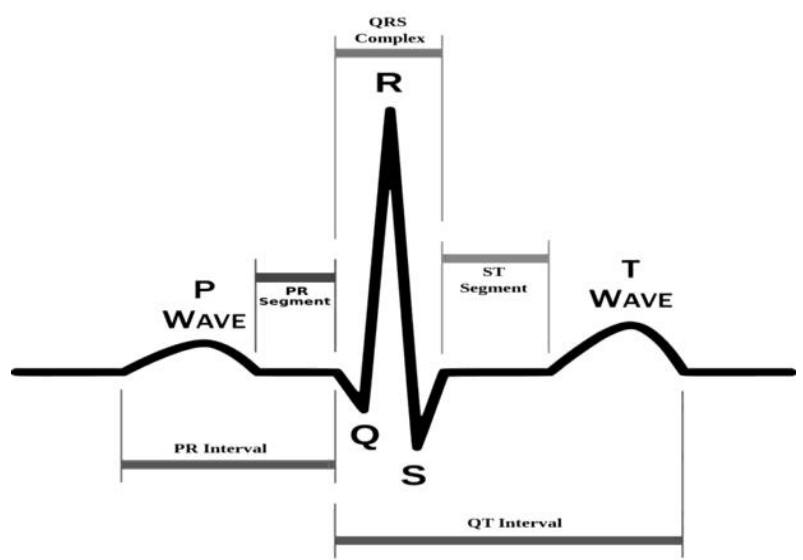

Figure 9. Standard ECG Signal

Table 2 shows normal ECG signal with different segment, such as $\mathrm{P}$ wave, PR interval, QRS Complex and also QT interval.

\begin{tabular}{lc}
\multicolumn{2}{c}{ Table2. Normal ECG Signal Data } \\
\hline Wave/In & Time Interval(ms) \\
\hline P wave & $0.06-0.11$ \\
PR Interval & $0.12-0.20$ \\
QRS complex & $0.08-0.12$ \\
QT interval & $0.36-0.44$ \\
\hline
\end{tabular}

Figure 10 shows the comparison of two ECG signals. Upper signal is normal person ECG signal. The bottom signal is the ECG signal with heart attack. From the figure, PR interval and also QRS Complex is concave. In this paper, main processing unit has a module to detect the ECG signal, also analyze the data in terms of different segment of ECG signal. Besides, the system is able to perform automatic car parking once it detect one of the segment of ECG signal is different from normal ECG signal.

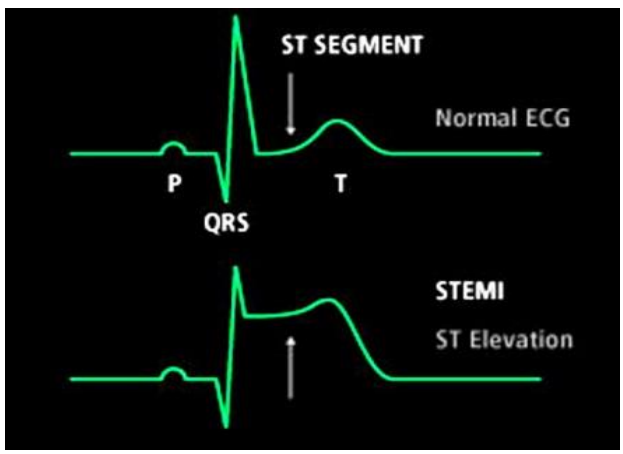

Figure. 10 Comparison between two ECG signals

\subsection{PWM Signal}

PWM is a modulation technique to encode a message into pulsing signal. In this paper, PWM signal used to control steering and also the speed of vehicles. Besides, ECG signal also been encoded into pulsing signal with specific frequency. Frequency is one of the main considerations when controlling PWM. The system will become unstable if the frequency didn't synchronize with the PWM. Several synchronize frequency modules were designed to control the frequency of several PWM including speed of vehicle control, steering and also the ECG signal as shown in Figure 11. 


\subsection{GPS and GSM}

GPS and GSM used for localization and send the coordinate of the host vehicle by SMS to hospital when a sudden heart attack happens to driver as shown in Figure 12.
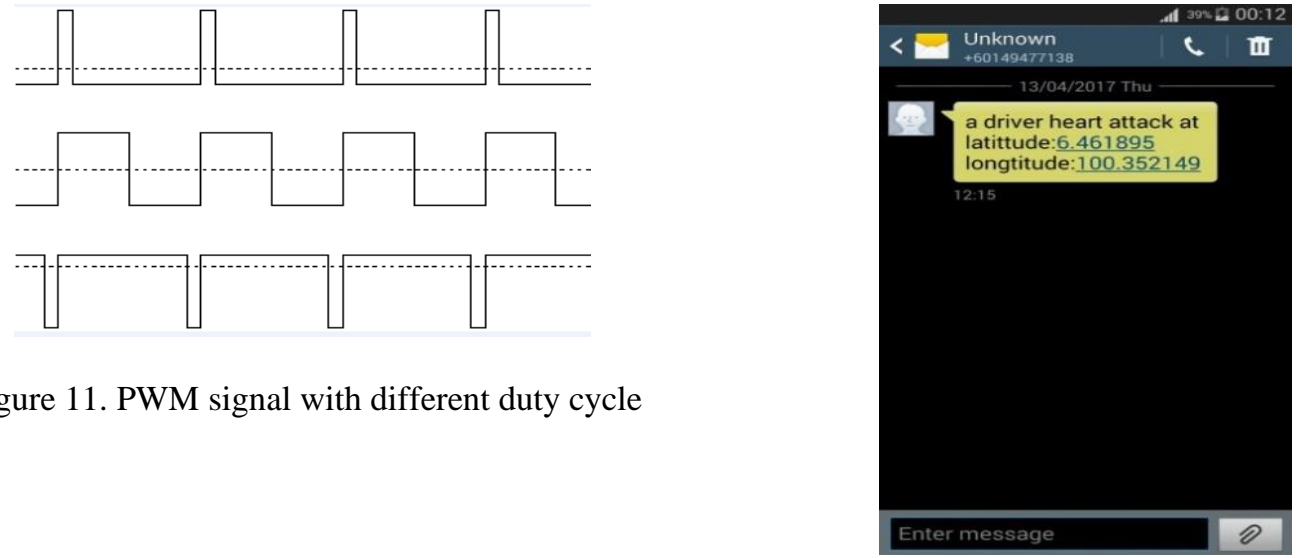

Figure. 12 GSM SMS

\section{DISCUSSION}

In this research, DE1-SoC board is chosen as a platform since it has a built-in ADC which consists of 8 channel number at a rate of 200KSPS. This platform is able to provide high speed data processing from multiple sensors and also concurrently task execution from multiple sensors. Reconfigurable of system and high speed data processing is one of the advantages for FPGA. Several on-chip modules are designed as control unit and processing unit. Besides, the system upgradeable without changing any hardware component in the future. On-chip module designed as much as possible to reduce latency of system because this project requires fast data processing and also precise decision making.

\section{CONCLUSION}

This paper introduces a new approach for current advanced driver assistance system which is automatic car parking system based on emergency condition including sudden heart attack and driver drowsiness from heart beat detector signal. The implementation is achieved high operating frequency up to $1.6 \mathrm{GHz}$ and the total of 1083 logic elements and 676 registers are consumed. In future, we can improve the robustness of drowsiness detection from facial expression and eye state of driver using a camera.

\section{ACKNOWLEDGEMENTS}

The authors would like to thank the Ministry of Education Malaysia (MOE) for providing the FRGS research grant (Ref: FRGS/2/2014/ICT06/UNIMAP/02/3) (Grant no. 9003-00474) and School of Computer and Communication Engineering, University Malaysia Perlis (UniMAP) for support.

\section{REFERENCES}

[1] Chowdhury M, and Dey K, "Intelligent transportation systems-a frontier for breaking boundaries of traditional academic engineering disciplines [Education]," IEEE Intell Transp Syst Mag, vol. 8, pp. 4-8, 2016.

[2] Bansal P, and Kockelman KM, "Forecasting Americans' longterm adoption of connected and autonomous vehicle technologies," In: Transportation research board 95th annual meeting, no. 16-1871, 2016.

[3] Saeed Asadi Bagloee, Madjid Tavana, Mohsen Asadi and Tracey Oliver, " Autonomous vehicles: challenges, opportunities, and future implications for transportation policies," Journal of Modern Transportation, vol. 24, no. 4, pp. 284-303, 2016.

[4] Han, S. J., and Choi, J., "Parking Space Recognition for Autonomous Valet Parking Using Height and Salient-Line Probability Maps," ETRI Journal, vol. 37, no. 6, pp. 1220-1230, 2015.

[5] Raksincharoensak, P., Hasegawa, T., and Nagai, M.. "Motion Planning and Control of Autonomous Driving Intelligence System Based on Risk Potential Optimization Framework," International Journal of Automotive Engineering, vol. 7, pp. 53-60, 2016. 
[6] Felipe Jiménez, José Eugenio Naranjo, José Javier Anaya, Fernando García, Aurelio Ponz and José María Armingol, "Advanced Driver Assistance System for Road Environments to Improve Safety and Efficiency," Transportation Research Procedia, vol. 14, pp. 2245-2254, 2016.

[7] Lam, A. Y., Leung, Y. W., and Chu, X.. "Autonomous-Vehicle Public Transportation System: Scheduling and Admission Control," IEEE Transactions on Intelligent Transportation Systems, vol. 17, no. 5, pp. 1210-1226, 2016.

[8] Mosquet, X., Andersen, M., and Arora, A., "A Roadmap to Safer Driving Through Advanced Driver Assistance Systems," Auto Tech Review, vol. 5, no. 7, pp. 20-25, 2016.

[9] Hamid, U. Z. A., Zamzuri, H., Rahman, M. A. A., \& Yahya and W. J., "A Safe-Distance Based Threat Assessment with Geometrical Based Steering Control for Vehicle Collision Avoidance," Journal of Telecommunication, Electronic and Computer Engineering (JTEC), vol. 8, no.2, pp. 53-58, 2016.

[10] C. N. Khac, J. H. Park and H. Y. Jung, "Detection of abnormal moving vehicles for intelligent driver assistance system," 2016 International Conference on Electronics, Information, and Communications (ICEIC), Da Nang, 2016, pp. 1-4.

[11] D. Tran, E. Tadesse, W. Sheng, Y. Sun, M. Liu and S. Zhang, "A driver assistance framework based on driver drowsiness detection," 2016 IEEE International Conference on Cyber Technology in Automation, Control, and Intelligent Systems (CYBER), Chengdu, 2016, pp. 173-178.

[12] D. Sunehra and K. Jhansi, "Implementation of microcontroller based driver assistance and vehicle safety monitoring system," 2015 International Conference on Information Processing (ICIP), Pune, 2016, pp. 423-428.

[13] T. Hwang, M. Kim, S. Hong and K. S. Park, "Driver drowsiness detection using the in-ear EEG," 2016 38th Annual International Conference of the IEEE Engineering in Medicine and Biology Society (EMBC), Orlando, FL, 2016, pp. 4646-4649.

[14] T. P. Nguyen, M. T. Chew and S. Demidenko, "Eye tracking system to detect driver drowsiness," 2015 6th International Conference on Automation, Robotics and Applications (ICARA), Queenstown, 2015, pp. 472-477.

[15] T. Nakamura, A. Maejima and S. Morishima, "Driver drowsiness estimation from facial expression features computer vision feature investigation using a CG model," 2014 International Conference on Computer Vision Theory and Applications (VISAPP), Lisbon, Portugal, 2015, pp. 207-214.

[16] L. Kurnianggoro, Wahyono, D. C. Hernández and K. H. Jo, "Camera and laser range finder fusion for real-time car detection," IECON 2014 - 40th Annual Conference of the IEEE Industrial Electronics Society, Dallas, TX, 2015, pp. 3419-3424.

\section{BIOGRAPHIES OF AUTHORS}

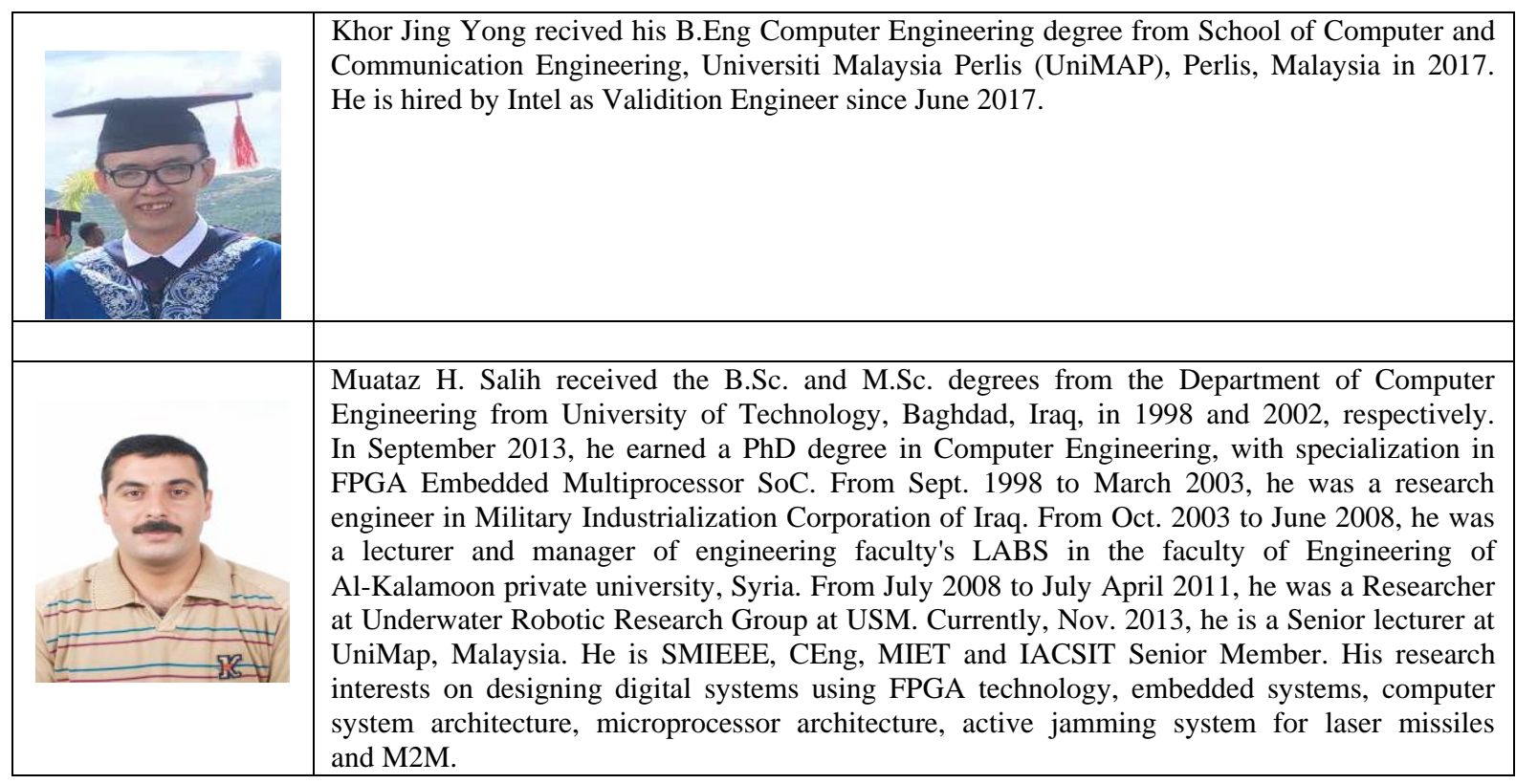

